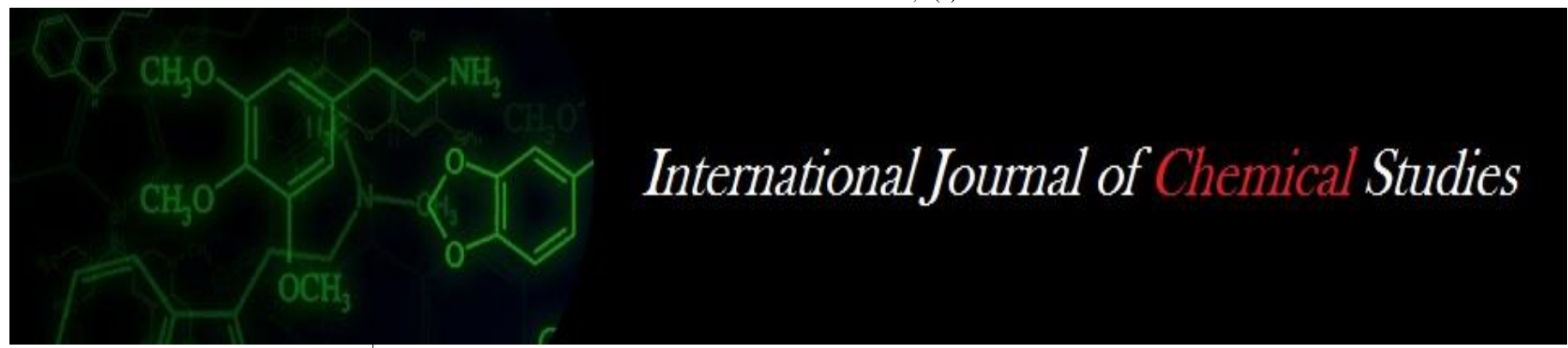

P-ISSN: 2349-8528

E-ISSN: 2321-4902

www.chemijournal.com

IJCS 2021; 9(1): 3645-3651

(C) 2021 IJCS

Received: 25-11-2020

Accepted: 20-12-2020

Surabhi VK

Department of Seed Science and

Technology, UAS, GKVK,

Bengaluru, Karnataka, India

Rame Gowda

Seed Technology Research Unit, NSP, UAS, GKVK, Bengaluru,

Karnataka, India

Nethra N

Seed Technology Research Unit, NSP, UAS, GKVK, Bengaluru,

Karnataka, India

Corresponding Author:

Surabhi VK

Department of Seed Science and

Technology, UAS, GKVK,

Bengaluru, Karnataka, India

\section{Influence of seed treatment with nanoparticles on seed quality and storability of pigeonpea cv. BRG-2}

\author{
Surabhi VK, Rame Gowda and Nethra N
}

DOI: https://doi.org/10.22271/chemi.2021.v9.i1ay.11799

\begin{abstract}
A laboratory experiment was conducted to study the influence of seed treatment with $\mathrm{ZnO}, \mathrm{Ag}$ and $\mathrm{SiO} 2$ nanoparticles and their bulk form on seed quality and storage potential of pigeonpea seeds. Results revealed significant effect of nanoparticles on seed quality and storability in pigeonpea. Among the treatment combinations, seeds treated with $\mathrm{SiO} 2 \mathrm{NPs} @ 250 \mathrm{mg}$ found to be superior for all the tested seed quality parameters viz., germination $(98,89$ and $80 \%)$, mean seedling dry weight $(46.45,44.95$ and 43.98mg), seedling vigour index-II (4553, 4016 and 3518), field emergence (94, 78 and 63\%), total dehydrogenase activity $(1.549,1.322$ and 1.196), lower electrical conductivity $(11.90,15.46$ and 41.29 $\mu \mathrm{S} / \mathrm{cm} / \mathrm{g}$ ) and reduced seed moisture content $(9.16,9.63$ and $10.09 \%)$ at 0,6 and 10 months of storage respectively. However, they were statistically on par with ZnO NPs @ $500 \mathrm{mg}$. The findings suggest the possibility of application of nanotechnology in enhancing seed quality and storability of pigeonpea.
\end{abstract}

Keywords: Nanoparticles, pigeonpea, seed quality and storability

\section{Introduction}

Pulses are an important group of crops that provide major source of dietary proteins complementing cereal proteins in human diets with essential amino acids, vitamins and minerals. Pulses are considered as 'Smart Food' as they are essential for food basket, containing 20-25 per cent of protein which is double the protein content of wheat and thrice that of rice thus providing nutritional and health benefit by addressing obesity, diabetes and malnutrition. Pigeonpea is one of the most important leguminous crops and is a rich source of protein and also rich in iron, iodine, essential amino acids like lycine, tyrocene, cystine and arginine (Tiwari and Shivhare 2018) ${ }^{[24]}$. Seed is a key input which decides the crop stand and yield under varied conditions. Poor of seed viability leads to storage loss in pulses which accounts to 30 to 70 per cent due to storage moulds, insect infestation especially bruchids and lipid peroxidation due to the production of reactive oxygen species leading to rapid deterioration. The storage potential of any seed depends on several factors like type of seed, period of storage, chemical composition, seed treatment, seed moisture, temperature and storage containers (Delouche and Baskin, 1973) ${ }^{[5]}$. The seed deterioration is inevitable, irreversible and inexorable but it can be delayed to some extent by proper management practices like preservation under controlled conditions and by advocating some seed invigouration trestments (Basu, 1994) ${ }^{[2]}$. Several strategies such as hydration and dehydration, halogenations, antioxidant treatments and seed disinfection technologies available to prolong the vigour and viability of seeds but does not allow storing of seeds for longer period and require immediate sowing and also may cause damage to seed coat. Thus, an improved seed invigouration treatments in both fresh and old seeds to improve seed viability and vigour during storage is essential. Nanotechnology is an emerging and fascinating field of science which permits novel applications in the field of agriculture and other disciplines. Preliminary studies showed the potential of nanoparticles (NPs) in improving seed germination and growth, plant protection, pathogen detection, and pesticide/herbicide residue detection (Khot $e t$ al., 2012) ${ }^{[8]}$. In seed science research, nanotechnology tools offers application of various nanoparticles for improvement of seed germination and related physiological parameters, nano membranes and nano polymer coating to enhance the storability of the seeds by incorporation of pesticides, nanosensors for better management of seed infestation during storage and 
incorporation of novel genes into seeds for specific trait (Chinnamuthu and Boopathi, 2009) [4]. Efficacy of nanoparticles is determined by their chemical composition, size, surface covering, reactivity and dose at which they are effective. The NPs are also known to donate electrons that pairs with the free radicals and thereby smother the impact of free radicals (Zheng et al., 2005) ${ }^{[25] .}$

Plants require micro elements in minute quantity for their growth and development, application of these elements in 'nano form' can be cost effective, besides reducing the usage of chemicals as an eco-friendly approach. Zinc ( $\mathrm{Zn})$ is essential micronutrients, required for the normal plant growth and development, important components of various enzymes responsible for many metabolic reactions. Increasing evidence suggests that zinc oxide nanoparticles increase plant growth and development in soybean (Sedghi et al., 2013) ${ }^{[15]}$. Silver (Ag) nanoparticles are among the most potential candidates for modulating the redox status of plants because of their ability to support electron exchange with $\mathrm{Fe} 2+$ and $\mathrm{Co} 3+$, the two elements that participate in several biological redox reactions (Sharma et al., 2012) ${ }^{[17]}$. Silicon is one of the most widespread macro elements, numerous field studies have shown that supplying crops with adequate plant available silicon can suppress plant disease, reduce insect attack, improve environmental stress tolerance and increase crop productivity. Nano silicon dioxide ( $\mathrm{SiO} 2)$ results in increased seed germination by providing better nutrients availability with adequate $\mathrm{pH}$ and conductivity to the growing medium in maize seeds (Suriyaprabha et al., 2012) [21]. However, from our earlier study on standardization of seed treatment protocol with nanoparticles for enhancing seed quality in pigeonpea (Surabhi et al., 2018) ${ }^{[20]}$ indicated dry seed treatment with $\mathrm{ZnO}, \mathrm{Ag}$ and $\mathrm{SiO} 2 \mathrm{NPs}$ found to have positive effect on seed quality in pigeonpea. Therefore, an effort was made to adopt an advanced third generation seed treatment with nanoparticles to enhance seed quality parameters and to prolong seed viability/shelf life in pigeonpea during storage under ambient conditions.

\section{Material and Methods}

The storage study was carried out at the Seed Technology Research Laboratory, All India Coordinated Research Project on Seed Technology Research Unit, National Seed Project (Crops), University of Agricultural Sciences, Gandhi Krishi Vignan Kendra, Bangalore during 2017-2018. Seeds of pigeonpea cv. BRG-2 and cloth bags were obtained from Seed Production Unit of National Seed Project (Crops), GKVK, Bangalore and dried to safe and uniform moisture level $(<9 \%)$. Zinc oxide $(\mathrm{ZnO})$, Silver $(\mathrm{Ag})$ and Silicon dioxide (SiO2) both bulk and nano forms were obtained from the
Sigma-Aldrich whose particle size was less than $100 \mathrm{~nm}$. The treatment combination of both nanoparticles (NPs) and bulk (B) viz., ZnO NPs @ 500 and 750 mg; ZnO bulk @ 750 and 1000 mg; Ag NPs@ 250 and 500 mg; Ag bulk@ 500 and 750 mg; SiO2 NPs@ 250 and 500 mg; SiO2 bulk @ 500 and $750 \mathrm{mg}$ were prepared for the experiment by thoroughly mixing the chemicals and seeds in a glass jar for even and uniform coating and the treated seeds were incubated for a short period to achieve equilibration. Thereafter these seeds were packed in cloth bag and stored under ambient conditions where the mean temperature was $23.42^{\circ} \mathrm{C}$ and mean relative humidity of $87.55 \%$ and $52.27 \%$ at $07.00 \mathrm{hr}$ and $14.00 \mathrm{hr}$, respectively for a period of ten months (June, 2017 - April, 2018). Further, various seed quality tests were conducted on monthly basis.

The seed moisture content $(\%)$, germination $(\%)$, mean seedling dry weight $(\mathrm{mg})$ and electrical conductivity $(\mu \mathrm{S} / \mathrm{cm} / \mathrm{g})$ were recorded as per ISTA (2013); the total dehydrogenase activity (A480 nm) as per Kittock and Law (1968) ${ }^{[9]}$; field emergence (\%) was also recorded by planting 100 seeds in three replications on a well prepared seed bed with adequate moisture and final count was taken on 12th day taking into account emergence of normal seedlings and expressed in percentage. The seedling vigour index-II was calculated as per Abdul-Baki and Anderson (1973) ${ }^{[1]}$.

\section{Seedling vigour index $\mathbf{- I I}=$ Germination $(\%)$ x Mean seedling dry weight (mg)}

The mean data of the storage experiment were statistically analyzed adopting completely randomized design with suitable ANOVA as outlined by Panse and Sukhatme (1985) [13]. The critical differences were calculated at five per cent level of probability wherever ' $F$ ' test was found significant for various seed quality parameters under the study.

\section{Results and Discussion}

Results of the present investigation showed that seed treatment with nanoparticles had significant influence on seed quality parameters. At the initial month of storage, no significant difference were observed in seed moisture content with SiO2 NPs @ 500 mg recording lowest seed moisture content $(9.08 \%)$ followed by SiO2 B @ $750 \mathrm{mg}(9.12 \%)$ and $\mathrm{SiO} 2 \mathrm{NPs} @ 250 \mathrm{mg}(9.16 \%)$ while the highest was recorded in ZnO NPs @ $750 \mathrm{mg}(9.28 \%)$. However, at the end of the storage period, $\mathrm{SiO} 2 \mathrm{NPs} @ 250 \mathrm{mg}$ recorded significantly lowest seed moisture content $(10.09 \%)$ followed by $\mathrm{SiO} 2 \mathrm{NPs}$ @ $500 \mathrm{mg}(10.19 \%)$, while the highest was recorded in control $(12.04 \%)$ which was significantly higher but not in safer limit (Table 1).

Table 1: Influence of seed treatment with nanoparticles on seed moisture content (\%) in pigeonpea cv. BRG-2 during storage

\begin{tabular}{|c|c|c|c|c|c|c|c|c|c|c|c|c|}
\hline \multirow{2}{*}{ Treatments } & \multicolumn{12}{|c|}{ Storage period in months (June, 2017 to April, 2018) } \\
\hline & 0 & 1 & 2 & 3 & 4 & 5 & 6 & 7 & 8 & 9 & 10 & Mean \\
\hline $\mathrm{T} 1$ & 9.20 & 9.28 & 9.57 & 9.88 & 10.04 & 10.10 & 10.45 & 10.90 & 11.05 & 11.71 & 12.04 & 10.38 \\
\hline $\mathrm{T} 2$ & 9.17 & 9.18 & 9.27 & 9.45 & 9.53 & 9.56 & 9.74 & 9.88 & 10.06 & 10.18 & 10.35 & 9.67 \\
\hline T3 & 9.28 & 9.30 & 9.40 & 9.68 & 9.80 & 9.84 & 10.00 & 10.45 & 10.77 & 10.88 & 11.03 & 10.04 \\
\hline $\mathrm{T} 4$ & 9.22 & 9.24 & 9.33 & 9.42 & 9.52 & 9.57 & 9.86 & 9.98 & 10.11 & 10.25 & 10.52 & 9.73 \\
\hline T5 & 9.25 & 9.26 & 9.35 & 9.45 & 9.58 & 9.61 & 9.98 & 10.09 & 10.18 & 10.30 & 10.59 & 9.79 \\
\hline T6 & 9.18 & 9.20 & 9.30 & 9.40 & 9.50 & 9.55 & 9.71 & 9.88 & 10.04 & 10.16 & 10.43 & 9.67 \\
\hline $\mathrm{T} 7$ & 9.22 & 9.26 & 9.37 & 9.46 & 9.57 & 9.59 & 9.92 & 10.02 & 10.14 & 10.28 & 10.56 & 9.76 \\
\hline T8 & 9.16 & 9.18 & 9.30 & 9.41 & 9.53 & 9.56 & 9.88 & 9.98 & 10.13 & 10.27 & 10.54 & 9.72 \\
\hline T9 & 9.16 & 9.20 & 9.32 & 9.42 & 9.51 & 9.54 & 9.85 & 9.95 & 10.08 & 10.21 & 10.50 & 9.70 \\
\hline T10 & 9.16 & 9.18 & 9.20 & 9.32 & 9.39 & 9.42 & 9.63 & 9.77 & 9.89 & 9.99 & 10.09 & 9.55 \\
\hline T11 & 9.08 & 9.10 & 9.18 & 9.23 & 9.31 & 9.35 & 9.55 & 9.83 & 9.97 & 10.08 & 10.19 & 9.53 \\
\hline
\end{tabular}




\begin{tabular}{|c|c|c|c|c|c|c|c|c|c|c|c|c|}
\hline T12 & 9.17 & 9.18 & 9.20 & 9.28 & 9.32 & 9.36 & 9.58 & 9.80 & 9.95 & 10.05 & 10.20 & 9.55 \\
\hline T13 & 9.12 & 9.16 & 9.19 & 9.27 & 9.30 & 9.33 & 9.59 & 9.84 & 9.99 & 10.10 & 10.24 & 9.56 \\
\hline Mean & 9.18 & 9.21 & 9.31 & 9.44 & 9.53 & 9.57 & 9.83 & 10.03 & 10.18 & 10.34 & 10.55 & 9.74 \\
\hline S.Em \pm & 0.040 & 0.007 & 0.010 & 0.003 & 0.006 & 0.004 & 0.005 & 0.005 & 0.005 & 0.005 & 0.003 & \\
\hline $\mathrm{CD}(0.05 \mathrm{P})$ & NS & 0.020 & 0.029 & 0.010 & 0.017 & 0.069 & 0.015 & 0.016 & 0.015 & 0.015 & 0.009 & \\
\hline CV (\%) & 0.750 & 0.131 & 0.188 & 0.061 & 0.108 & 0.011 & 0.094 & 0.094 & 0.088 & 0.086 & 0.053 & \\
\hline
\end{tabular}

T1 - Control, T2 - ZnO NPs @ 500 mg, T3 - ZnO NPs @ 750 mg, T4 - ZnO B @ 750 mg, T5 - ZnO B @ 1000 mg, T6 - Ag NPs @ 250 mg, T7 - Ag NPs @ 500 mg, T8 - Ag B @ 500 mg, T9 - Ag B @ 750 mg, T10 - SiO2 NPs@ 250 mg, T11 - SiO2 NPs@ 500 mg, T12 - SiO2 B@ 500 mg, T13 - SiO2 B @ 750 mg

The moisture content of seed increased as the storage period advanced which may be due fluctuations in relative humidity and temperature in the surrounding environmental conditions since the seeds were stored in the cloth bag that might have result in and metabolic release of water during respiration. The fluctuation of moisture content was significantly higher in untreated seeds compared to seed treatment with nanoparticles. Probably the nanoparticles might have acted as physical barrier, which is assumed to restrict the movement of water vapour in and out of the treated seeds and hence, reduced the fluctuation of moisture content during the storage. Another major beneficial effect of silicon dioxide might be related to its hydrophilicity thus maintaining seed moisture content in safer limit throughout the storage period. Similar observations were also reported by Korishettar et al. (2017) ${ }^{[10]}$ on $\mathrm{Zn}$ and $\mathrm{Fe}$ nanoparticles (NPs) in pigeonpea.

A significant influence on seed germination percentage by seed treatment with nanoparticles was observed during storage period of ten months (Table 2). In the initial month of storage, highest germination was recorded in $\mathrm{SiO} 2 \mathrm{NPs} @$ $250 \mathrm{mg}(98 \%)$ and it was statistically on par with Ag NPs @ 250 mg (97\%), SiO2 B @ 500 mg (97\%) and ZnO NPs@ $500 \mathrm{mg}(96 \%)$, while the lowest germination was recorded in control (93\%). At the end of ten months of storage, highest germination was recorded in SiO2 NPs @ $250 \mathrm{mg}(80 \%)$ which was on par with ZnO NPs @ 500 mg (79\%) followed by $\mathrm{SiO} 2$ NPs @ $500 \mathrm{mg}(77 \%)$ and lowest germination was recorded in control (61\%). The reason might be that nanoparticles would induce oxidation-reduction reactions via superoxide ion radicals during germination, resulting the quenching of free radicals in the aged seeds. In turn, oxygen produced in such process could also be used for respiration, which would further promote germination (Zheng et al., $2005)^{[25]}$. Exogenous application of silicon may improve seed germination and seedling growth by enhancing antioxidant defense and improvement in Fe nutrition (Shi et al., 2014) ${ }^{[18]}$. Similar findings were reported by Tejaswini et al. (2019) ${ }^{[23]}$ in groundnut.

Table 2: Influence of seed treatment with nanoparticles on germination (\%) in pigeonpea cv. BRG-2 during storage

\begin{tabular}{|c|c|c|c|c|c|c|c|c|c|c|c|c|}
\hline \multirow{2}{*}{ Treatments } & \multicolumn{12}{|c|}{ Storage period in months (June, 2017 to April, 2018) } \\
\hline & $\mathbf{0}$ & 1 & 2 & 3 & 4 & 5 & 6 & 7 & 8 & 9 & 10 & Mean \\
\hline $\mathrm{T} 1$ & 93 & 91 & 89 & 87 & 84 & 82 & 79 & 77 & 73 & 68 & 61 & 80 \\
\hline $\mathrm{T} 2$ & 96 & 95 & 93 & 92 & 91 & 90 & 87 & 85 & 83 & 81 & 79 & 88 \\
\hline T3 & 95 & 94 & 92 & 91 & 89 & 87 & 85 & 82 & 80 & 77 & 74 & 86 \\
\hline $\mathrm{T} 4$ & 96 & 94 & 91 & 89 & 87 & 85 & 83 & 80 & 79 & 74 & 71 & 84 \\
\hline $\mathrm{T} 5$ & 95 & 92 & 89 & 87 & 85 & 84 & 81 & 78 & 75 & 72 & 69 & 82 \\
\hline T6 & 97 & 94 & 91 & 90 & 88 & 86 & 83 & 81 & 78 & 76 & 73 & 85 \\
\hline $\mathrm{T} 7$ & 94 & 94 & 90 & 88 & 84 & 83 & 81 & 79 & 77 & 74 & 70 & 83 \\
\hline $\mathrm{T} 8$ & 95 & 94 & 91 & 89 & 86 & 84 & 82 & 80 & 76 & 72 & 68 & 83 \\
\hline T9 & 93 & 91 & 89 & 86 & 85 & 82 & 80 & 78 & 74 & 71 & 68 & 82 \\
\hline $\mathrm{T} 10$ & 98 & 96 & 94 & 93 & 92 & 91 & 89 & 86 & 83 & 82 & 80 & 89 \\
\hline T11 & 95 & 95 & 93 & 91 & 90 & 89 & 86 & 84 & 82 & 80 & 77 & 87 \\
\hline T12 & 97 & 94 & 92 & 90 & 89 & 87 & 84 & 83 & 81 & 78 & 75 & 86 \\
\hline T13 & 94 & 93 & 91 & 89 & 88 & 86 & 82 & 80 & 77 & 75 & 73 & 84 \\
\hline Mean & 95 & 94 & 91 & 89 & 87 & 86 & 83 & 81 & 78 & 75 & 72 & 85 \\
\hline S.Em \pm & 0.64 & 0.65 & 0.51 & 0.42 & 0.37 & 0.32 & 0.33 & 0.38 & 0.40 & 0.38 & 0.32 & \\
\hline $\mathrm{CD}(0.05 \mathrm{P})$ & 1.86 & 1.88 & 1.47 & 1.23 & 1.07 & 0.93 & 0.97 & 1.11 & 1.17 & 1.11 & 0.93 & \\
\hline $\mathrm{CV}(\%)$ & 1.16 & 1.20 & 0.96 & 0.82 & 0.73 & 0.65 & 0.69 & 0.81 & 0.89 & 0.88 & 0.77 & \\
\hline
\end{tabular}

T1 - Control, T2 - ZnO NPs @ 500 mg, T3 - ZnO NPs @ 750 mg, T4 - ZnO B @ 750 mg, T5 - ZnO B @ 1000 mg, T6 - Ag NPs @ 250 mg, T7 - Ag NPs@ 500 mg, T8 - Ag B @ 500 mg, T9 - Ag B @ 750 mg, T10 - SiO2 NPs@ 250 mg, T11 - SiO2 NPs @ 500 mg, T12 - SiO2 B @ 500 mg, T13 - SiO2 B @ 750 mg

The results on mean seedling dry weight (mg) were significantly influenced by seed treatment with nanoparticles during storage. Irrespective of the seed treatments, mean seedling dry weight decreased gradually with the storage period. At the end of the storage period, SiO2 NPs@ $250 \mathrm{mg}$ and ZnO NPs @ $500 \mathrm{mg}$ recorded higher seedling dry weight (43.98 and $43.93 \mathrm{mg}$, respectively) compared to control
(36.25 mg) (Table 3). Pandey et al. (2010) ${ }^{[12]}$ reported that $\mathrm{ZnO}$ NPs increased the level of IAA in the roots (sprouts) and thereby an increase in the growth rate in Cicer arietinum. Besides increased synthesis and activity of hydrolytic enzymes resulted in increased dry matter production. Similar observations were also made by Tahir et al. (2010) ${ }^{[2]}$ where wheat biomass increased with the application of silicon. 
Table 3: Influence of seed treatment with nanoparticles on mean seedling dry weight (mg/seedling) in pigeonpea cv. BRG-2 during storage

\begin{tabular}{|c|c|c|c|c|c|c|c|c|c|c|c|c|c|}
\hline \multirow{2}{*}{ Treatments } & \multicolumn{10}{|c|}{ Storage period in months (June, 2017 to April, 2018) } \\
\cline { 2 - 13 } & $\mathbf{0}$ & $\mathbf{1}$ & $\mathbf{2}$ & $\mathbf{3}$ & $\mathbf{4}$ & $\mathbf{5}$ & $\mathbf{6}$ & $\mathbf{7}$ & $\mathbf{8}$ & $\mathbf{9}$ & $\mathbf{1 0}$ & Mean \\
\hline T1 & 38.52 & 38.07 & 37.88 & 37.43 & 37.36 & 37.05 & 36.93 & 36.76 & 36.60 & 36.44 & 36.25 & 37.21 \\
\hline T2 & 46.91 & 46.70 & 45.88 & 45.52 & 45.45 & 45.13 & 44.94 & 44.75 & 44.46 & 44.20 & 43.93 & 45.26 \\
\hline T3 & 43.67 & 43.42 & 43.10 & 42.58 & 42.48 & 42.17 & 42.98 & 42.75 & 42.54 & 42.35 & 42.20 & 42.75 \\
\hline T4 & 41.75 & 40.07 & 39.93 & 39.67 & 39.60 & 39.45 & 39.17 & 38.95 & 38.76 & 38.55 & 38.33 & 39.48 \\
\hline T5 & 42.00 & 41.65 & 41.32 & 41.11 & 41.04 & 40.78 & 40.52 & 40.32 & 40.16 & 39.93 & 39.76 & 40.78 \\
\hline T6 & 42.10 & 41.82 & 41.53 & 41.20 & 41.12 & 40.90 & 40.76 & 40.55 & 40.21 & 40.04 & 39.86 & 40.92 \\
\hline T7 & 43.23 & 42.92 & 42.76 & 42.46 & 42.38 & 42.15 & 41.90 & 41.78 & 41.54 & 41.32 & 41.16 & 42.15 \\
\hline T8 & 41.30 & 40.75 & 40.51 & 40.31 & 40.27 & 40.03 & 39.86 & 39.63 & 39.50 & 39.29 & 39.12 & 40.05 \\
\hline T9 & 41.05 & 40.25 & 40.09 & 39.86 & 39.82 & 39.58 & 39.36 & 39.20 & 38.93 & 38.76 & 38.53 & 39.58 \\
\hline T10 & 46.45 & 46.15 & 45.97 & 45.60 & 45.47 & 45.17 & 44.95 & 44.77 & 44.48 & 44.23 & 43.98 & 45.20 \\
\hline T11 & 44.00 & 43.85 & 43.66 & 43.34 & 43.28 & 43.01 & 42.83 & 42.78 & 42.56 & 42.39 & 42.26 & 43.09 \\
\hline T12 & 42.57 & 42.13 & 41.95 & 41.73 & 41.65 & 41.33 & 41.15 & 40.96 & 40.77 & 40.51 & 40.31 & 41.37 \\
\hline T13 & 42.60 & 42.41 & 42.25 & 41.09 & 40.98 & 40.66 & 40.46 & 40.29 & 40.06 & 39.85 & 39.68 & 40.94 \\
\hline Mean & 42.78 & 42.32 & 42.06 & 41.68 & 41.61 & 41.34 & 41.22 & 41.04 & 40.81 & 40.61 & 40.41 & 41.44 \\
\hline S.Em \pm & 1.02 & 0.05 & 0.02 & 0.09 & 0.01 & 0.01 & 0.01 & 0.01 & 0.03 & 0.01 & 0.01 & \\
\hline CD $(0.05 P)$ & 2.97 & 0.15 & 0.06 & 0.27 & 0.02 & 0.02 & 0.02 & 0.02 & 0.09 & 0.03 & 0.02 \\
\hline CV $(\%)$ & 4.14 & 0.21 & 0.09 & 0.38 & 0.02 & 0.02 & 0.02 & 0.02 & 0.13 & 0.02 & 0.02 & \\
\hline Cont
\end{tabular}

T1 - Control, T2 - ZnO NPs @ 500 mg, T3 - ZnO NPs @ 750 mg, T4 - ZnO B @ 750 mg, T5 - ZnO B @ 1000 mg, T6 - Ag NPs @ 250 mg, T7 - Ag NPs@500 mg, T8 - Ag B @ 500 mg, T9 - Ag B @ 750 mg, T10 - SiO2 NPs@ 250 mg, T11 - SiO2 NPs@ 500 mg, T12 - SiO2 B @ 500 $\mathrm{mg}, \mathrm{T} 13$ - SiO2 B @ $750 \mathrm{mg}$

Irrespective of the treatments, seedling vigour index-II and field emergence percentage gradually declined with the storage period advancement. At the end of ten months of storage, highest seedling vigour index-II and field emergence (3518 and 63\%, respectively) recorded in SiO2 NPs @ 250 $\mathrm{mg}$ followed by ZnO NPs @ $500 \mathrm{mg}$ (3485 and 61\%, respectively) compared to control (2224 and 45\%, respectively) (Table $4 \& 5$ ). This could be ascribed to the beneficial effects of these NPs in repairing of damaged vital cell organelles, synthesis and activation of essential enzymes during germination and counteraction of lipid peroxidation and minimization of free radical reactions. Senthil Kumar et al. (2011) ${ }^{[16]}$ reported that black gram seeds treated with $\mathrm{ZnO}$ nano rods and ZVI NPs enhanced the physiological and biochemical properties resulting in improved vigour and viability of aged seeds. Similar results were reported by Krishna Shyla and Natarajan (2014) in groundnut; Korishettar et al. (2017) ${ }^{[10]}$ on $\mathrm{Zn}$ and Fe nanoparticles (NPs) in pigeonpea.

Table 4: Influence of seed treatment with nanoparticles on seedling vigour index-II in pigeonpea cv. BRG-2 during storage

\begin{tabular}{|c|c|c|c|c|c|c|c|c|c|c|c|c|}
\hline \multirow{2}{*}{ Treatments } & \multicolumn{10}{|c|}{ Storage period in months (June, 2017 to April, 2018) } \\
\cline { 2 - 13 } & $\mathbf{0}$ & $\mathbf{1}$ & $\mathbf{2}$ & $\mathbf{3}$ & $\mathbf{4}$ & $\mathbf{5}$ & $\mathbf{6}$ & $\mathbf{7}$ & $\mathbf{8}$ & $\mathbf{9}$ & $\mathbf{1 0}$ & Mean \\
\hline $\mathrm{T} 1$ & 3569 & 3464 & 3371 & 3269 & 3138 & 3026 & 2930 & 2831 & 2684 & 2466 & 2224 & 2997 \\
\hline $\mathrm{T} 2$ & 4519 & 4436 & 4252 & 4188 & 4121 & 4047 & 3925 & 3819 & 3690 & 3595 & 3485 & 4007 \\
\hline $\mathrm{T} 3$ & 4133 & 4082 & 3965 & 3861 & 3795 & 3683 & 3639 & 3519 & 3389 & 3247 & 3109 & 3675 \\
\hline $\mathrm{T} 4$ & 4004 & 3766 & 3633 & 3531 & 3445 & 3353 & 3238 & 3129 & 3049 & 2852 & 2709 & 3337 \\
\hline $\mathrm{T} 5$ & 3991 & 3832 & 3691 & 3591 & 3475 & 3439 & 3269 & 3159 & 3025 & 2862 & 2730 & 3369 \\
\hline $\mathrm{T} 6$ & 4084 & 3945 & 3779 & 3694 & 3605 & 3504 & 3397 & 3271 & 3150 & 3056 & 2923 & 3492 \\
\hline $\mathrm{T} 7$ & 4064 & 4035 & 3834 & 3750 & 3574 & 3485 & 3380 & 3315 & 3185 & 3058 & 2895 & 3507 \\
\hline T8 & 3937 & 3817 & 3687 & 3588 & 3450 & 3376 & 3282 & 3171 & 3002 & 2816 & 2673 & 3345 \\
\hline T9 & 3818 & 3663 & 3581 & 3441 & 3371 & 3259 & 3136 & 3071 & 2881 & 2765 & 2607 & 3236 \\
\hline T10 & 4553 & 4446 & 4321 & 4226 & 4183 & 4096 & 4016 & 3865 & 3707 & 3642 & 3518 & 4052 \\
\hline T11 & 4165 & 4165 & 4075 & 3944 & 3881 & 3842 & 3669 & 3608 & 3504 & 3406 & 3240 & 3773 \\
\hline T12 & 4131 & 3960 & 3859 & 3769 & 3721 & 3609 & 3443 & 3386 & 3289 & 3174 & 3010 & 3577 \\
\hline T13 & 4018 & 3958 & 3831 & 3671 & 3593 & 3511 & 3331 & 3236 & 3098 & 2975 & 2884 & 3464 \\
\hline Mean & 4076 & 3967 & 3837 & 3732 & 3642 & 3556 & 3435 & 3337 & 3204 & 3070 & 2924 & 3525 \\
\hline S.Em \pm & 96.79 & 29.39 & 21.51 & 21.13 & 15.13 & 13.16 & 13.81 & 15.43 & 16.03 & 15.49 & 12.74 & \\
\hline CD (0.05P) & 281.37 & 85.45 & 62.51 & 61.43 & 43.99 & 38.27 & 40.15 & 44.85 & 46.60 & 45.03 & 37.04 \\
\hline CV (\%) & 4.11 & 1.28 & 0.97 & 0.98 & 0.72 & 0.64 & 0.70 & 0.80 & 0.87 & 0.87 & 0.75 & \\
\hline
\end{tabular}

T1 - Control, T2 - ZnO NPs @ 500 mg, T3 - ZnO NPs @ 750 mg, T4 - ZnO B @ 750 mg, T5 - ZnO B @ 1000 mg, T6 - Ag NPs @ 250 mg, T7 - Ag NPs@ 500 mg, T8 - Ag B @ 500 mg, T9 - Ag B @ 750 mg, T10 - SiO2 NPs@ 250 mg, T11 - SiO2 NPs@ 500 mg, T12 - SiO2 B @ 500 $\mathrm{mg}, \mathrm{T} 13$ - SiO2 B @ $750 \mathrm{mg}$ 
Table 5: Influence of seed treatment with nanoparticles on field emergence (\%) in pigeonpea cv. BRG-2 during storage

\begin{tabular}{|c|c|c|c|c|c|c|c|c|c|c|c|c|c|}
\hline \multirow{2}{*}{ Treatments } & \multicolumn{10}{|c|}{ Storage period in months (June, 2017 to April, 2018) } \\
\cline { 2 - 14 } & $\mathbf{0}$ & $\mathbf{1}$ & $\mathbf{2}$ & $\mathbf{3}$ & $\mathbf{4}$ & $\mathbf{5}$ & $\mathbf{6}$ & $\mathbf{7}$ & $\mathbf{8}$ & $\mathbf{9}$ & $\mathbf{1 0}$ & Mean \\
\hline T1 & 90 & 89 & 85 & 82 & 80 & 77 & 69 & 63 & 57 & 53 & 45 & 72 \\
\hline T2 & 94 & 92 & 90 & 88 & 85 & 80 & 78 & 74 & 69 & 65 & 61 & 80 \\
\hline T3 & 88 & 86 & 84 & 84 & 82 & 78 & 76 & 74 & 68 & 64 & 58 & 77 \\
\hline T4 & 90 & 87 & 86 & 83 & 81 & 76 & 76 & 73 & 66 & 63 & 57 & 76 \\
\hline T5 & 87 & 86 & 83 & 81 & 79 & 75 & 72 & 66 & 62 & 57 & 52 & 73 \\
\hline T6 & 92 & 90 & 88 & 85 & 83 & 78 & 75 & 69 & 65 & 60 & 54 & 76 \\
\hline T7 & 86 & 85 & 84 & 81 & 78 & 74 & 72 & 67 & 64 & 58 & 53 & 73 \\
\hline T8 & 91 & 89 & 87 & 83 & 81 & 77 & 74 & 69 & 65 & 59 & 55 & 75 \\
\hline T9 & 86 & 84 & 81 & 80 & 78 & 74 & 73 & 68 & 62 & 56 & 52 & 72 \\
\hline T10 & 94 & 93 & 91 & 89 & 87 & 82 & 78 & 75 & 70 & 67 & 63 & 81 \\
\hline T11 & 89 & 89 & 86 & 84 & 82 & 77 & 76 & 74 & 69 & 64 & 60 & 77 \\
\hline T12 & 92 & 90 & 88 & 85 & 83 & 76 & 74 & 70 & 67 & 63 & 56 & 77 \\
\hline T13 & 88 & 87 & 85 & 82 & 80 & 75 & 72 & 68 & 61 & 56 & 52 & 73 \\
\hline Mean & 90 & 88 & 86 & 83 & 81 & 77 & 74 & 70 & 65 & 60 & 55 & 76 \\
\hline S.Em \pm & 0.89 & 0.55 & 0.56 & 0.42 & 0.33 & 0.38 & 0.36 & 0.38 & 0.40 & 0.32 & 0.33 & \\
\hline CD $(0.05 P)$ & 2.58 & 1.59 & 1.62 & 1.23 & 0.97 & 1.11 & 1.04 & 1.11 & 1.17 & 0.93 & 0.97 \\
\hline CV $(\%)$ & 1.71 & 1.07 & 1.13 & 0.88 & 0.71 & 0.86 & 0.84 & 0.94 & 1.07 & 0.92 & 1.05 & \\
\hline
\end{tabular}

T1 - Control, T2 - ZnO NPs @ 500 mg, T3 - ZnO NPs @ 750 mg, T4 - ZnO B @ 750 mg, T5 - ZnO B @ 1000 mg, T6 - Ag NPs @ 250 mg, T7 - Ag NPs@ 500 mg, T8 - Ag B @ 500 mg, T9 - Ag B @ 750 mg, T10 - SiO2 NPs@ 250 mg, T11 - SiO2 NPs @ 500 mg, T12 - SiO2 B @ 500 $\mathrm{mg}, \mathrm{T} 13$ - SiO2 B @ $750 \mathrm{mg}$

A linear increase in the electrical conductivity of seed leachate $(\mu \mathrm{S} / \mathrm{cm} / \mathrm{g})$ with increasing storage period was observed (Table 6). At the end of storage period, control recorded highest electrical conductivity $(55.42 \mu \mathrm{S} / \mathrm{cm} / \mathrm{g})$ while, SiO2 NPs@250 mg recorded lowest electrical conductivity $(41.29 \mu \mathrm{S} / \mathrm{cm} / \mathrm{g})$ followed by ZnO NPs @ 500 $\mathrm{mg}(41.78 \mu \mathrm{S} / \mathrm{cm} / \mathrm{g})$. The results depicts that the thin layer of nanoparticles over seeds surface maintains the seed coat integrity thereby reduces the leakage of solutes from the seeds. Sahebi et al. (2015) ${ }^{[14]}$ reported that silicon increases the plasma membrane integrity by providing more stable lipids involved in their cell membrane. However, the EC of untreated seeds of pegionpea was enormous at the end of seed storage which is an indicative factor of seed deterioration.

Table 6: Influence of seed treatment with nanoparticles on electrical conductivity of seed leachate $(\mu \mathrm{S} / \mathrm{cm} / \mathrm{g})$ in pigeonpea cv. BRG- 2 during storage

\begin{tabular}{|c|c|c|c|c|c|c|c|c|c|c|c|c|}
\hline \multirow{2}{*}{ Treatments } & \multicolumn{10}{|c|}{ Storage period in months (June, 2017 to April, 2018) } \\
\cline { 2 - 13 } & $\mathbf{0}$ & $\mathbf{1}$ & $\mathbf{2}$ & $\mathbf{3}$ & $\mathbf{4}$ & $\mathbf{5}$ & $\mathbf{6}$ & $\mathbf{7}$ & $\mathbf{8}$ & $\mathbf{9}$ & $\mathbf{1 0}$ & Mean \\
\hline T1 & 13.52 & 14.12 & 14.82 & 15.05 & 15.60 & 16.18 & 22.44 & 29.87 & 38.41 & 49.21 & 55.42 & 25.88 \\
\hline T2 & 12.12 & 12.84 & 13.03 & 13.44 & 13.68 & 13.77 & 16.66 & 20.55 & 26.89 & 35.43 & 41.78 & 20.02 \\
\hline T3 & 13.63 & 13.91 & 14.10 & 14.47 & 14.92 & 14.98 & 18.74 & 22.43 & 30.68 & 40.33 & 47.34 & 22.32 \\
\hline T4 & 13.49 & 14.01 & 14.23 & 14.57 & 15.03 & 15.16 & 19.55 & 25.62 & 32.98 & 41.57 & 47.98 & 23.11 \\
\hline T5 & 14.35 & 14.82 & 15.06 & 15.37 & 15.58 & 15.78 & 19.87 & 25.77 & 33.27 & 42.18 & 48.24 & 23.66 \\
\hline T6 & 13.74 & 14.33 & 14.68 & 14.98 & 15.26 & 15.56 & 18.61 & 24.59 & 33.97 & 42.78 & 48.66 & 23.38 \\
\hline T7 & 13.15 & 13.74 & 13.98 & 14.25 & 14.56 & 14.67 & 17.41 & 24.36 & 34.13 & 43.05 & 49.43 & 22.98 \\
\hline T8 & 12.43 & 13.08 & 13.45 & 13.79 & 14.05 & 14.18 & 17.29 & 24.23 & 34.10 & 43.44 & 49.77 & 22.71 \\
\hline T9 & 13.51 & 13.73 & 13.87 & 14.07 & 14.29 & 14.39 & 17.45 & 23.98 & 32.93 & 41.98 & 48.13 & 22.58 \\
\hline T10 & 11.90 & 11.96 & 12.08 & 12.44 & 12.66 & 12.77 & 15.46 & 20.26 & 26.45 & 35.24 & 41.29 & 19.32 \\
\hline T11 & 12.32 & 12.46 & 12.78 & 12.97 & 13.11 & 13.25 & 16.98 & 20.77 & 27.02 & 36.15 & 46.98 & 20.44 \\
\hline T12 & 12.17 & 12.58 & 12.88 & 13.05 & 13.42 & 13.55 & 17.24 & 23.56 & 32.45 & 41.28 & 47.45 & 21.78 \\
\hline T13 & 12.67 & 12.76 & 12.91 & 13.11 & 13.53 & 13.64 & 17.39 & 23.77 & 32.77 & 41.96 & 48.09 & 22.05 \\
\hline Mean & 13.00 & 13.41 & 13.68 & 13.97 & 14.28 & 14.45 & 18.08 & 23.83 & 32.00 & 41.12 & 47.74 & 22.32 \\
\hline S.Em \pm & 0.16 & 0.04 & 0.02 & 0.01 & 0.01 & 0.01 & 0.02 & 0.01 & 0.01 & 0.01 & 0.01 & \\
\hline CD $(0.05 P)$ & 0.46 & 0.13 & 0.05 & 0.02 & 0.02 & 0.02 & 0.05 & 0.02 & 0.02 & 0.02 & 0.02 \\
\hline CV $(\%)$ & 2.09 & 0.56 & 0.20 & 0.07 & 0.09 & 0.06 & 0.15 & 0.04 & 0.03 & 0.02 & 0.01 \\
\hline Cont
\end{tabular}

T1 - Control, T2 - ZnO NPs @ 500 mg, T3 - ZnO NPs @ 750 mg, T4 - ZnO B @ 750 mg, T5 - ZnO B @ 1000 mg, T6 - Ag NPs @ 250 mg, T7 - Ag NPs@ 500 mg, T8 - Ag B @ 500 mg, T9 - Ag B @ 750 mg, T10 - SiO2 NPs@ 250 mg, T11 - SiO2 NPs @ 500 mg, T12 - SiO2 B @ 500 $\mathrm{mg}, \mathrm{T} 13$ - SiO2 B @ $750 \mathrm{mg}$

Seed treatment with nanoparticles on physiological parameter like total dehydrogenase activity (A480 nm) showed elevated effect. A significant variation in dehydrogenase activity (TDH) was observed in seeds treated with $\mathrm{ZnO}, \mathrm{Ag}$ and $\mathrm{SiO} 2$ nanoparticles during the initial months of storage and gradual decline in the activity was also observed with advancement of storage period irrespective of the treatments (Table 7). Results showed that $\mathrm{SiO} 2 \mathrm{NPs} @ 250 \mathrm{mg}$ recorded significantly higher dehydrogenase activity (1.549) followed by SiO2 NPs @ 500 mg (1.434) and ZnO NPs @ 500 mg (1.432) while the lowest was recorded in ZnO B @ 1000 mg (1.138). At the end of the storage period, SiO2 NPs @ $250 \mathrm{mg}$ recorded significantly higher dehydrogenase activity (1.196) followed by $\mathrm{SiO} 2$ NPs @ 500 mg (1.191) and ZnO NPs@ 500 mg (1.164) while the lowest was recorded in bulk form of $\mathrm{ZnO} @$ $1000 \mathrm{mg}(0.924)$ followed by control (0.925). 
Table 7: Influence of seed treatment with nanoparticles on total dehydrogenase activity (A480nm) in pigeonpea cv. BRG-2 during storage

\begin{tabular}{|c|c|c|c|c|c|c|c|c|c|c|c|c|c|}
\hline \multirow{2}{*}{ Treatments } & \multicolumn{10}{|c|}{ Storage period in months (June, 2017 to April, 2018) } \\
\cline { 2 - 14 } & $\mathbf{0}$ & $\mathbf{1}$ & $\mathbf{2}$ & $\mathbf{3}$ & $\mathbf{4}$ & $\mathbf{5}$ & $\mathbf{6}$ & $\mathbf{7}$ & $\mathbf{8}$ & $\mathbf{9}$ & $\mathbf{1 0}$ & Mean \\
\hline T1 & 1.288 & 1.259 & 1.228 & 1.194 & 1.172 & 1.145 & 1.120 & 1.077 & 1.025 & 0.984 & 0.925 & 1.129 \\
\hline T2 & 1.432 & 1.417 & 1.395 & 1.352 & 1.322 & 1.316 & 1.298 & 1.254 & 1.228 & 1.197 & 1.164 & 1.307 \\
\hline T3 & 1.407 & 1.397 & 1.367 & 1.346 & 1.320 & 1.306 & 1.254 & 1.228 & 1.198 & 1.155 & 1.138 & 1.283 \\
\hline T4 & 1.176 & 1.152 & 1.140 & 1.113 & 1.094 & 1.076 & 1.054 & 1.039 & 1.025 & 0.994 & 0.967 & 1.075 \\
\hline T5 & 1.138 & 1.108 & 1.097 & 1.057 & 1.036 & 1.022 & 1.011 & 0.996 & 0.973 & 0.943 & 0.924 & 1.028 \\
\hline T6 & 1.368 & 1.338 & 1.323 & 1.298 & 1.256 & 1.233 & 1.214 & 1.196 & 1.170 & 1.157 & 1.134 & 1.244 \\
\hline T7 & 1.237 & 1.203 & 1.197 & 1.165 & 1.122 & 1.110 & 1.096 & 1.063 & 1.032 & 1.011 & 0.990 & 1.111 \\
\hline T8 & 1.153 & 1.127 & 1.103 & 1.076 & 1.046 & 1.029 & 1.006 & 0.984 & 0.971 & 0.954 & 0.936 & 1.035 \\
\hline T9 & 1.212 & 1.193 & 1.176 & 1.134 & 1.108 & 1.094 & 1.056 & 1.026 & 0.997 & 0.974 & 0.954 & 1.084 \\
\hline T10 & 1.549 & 1.434 & 1.415 & 1.398 & 1.356 & 1.345 & 1.322 & 1.298 & 1.256 & 1.228 & 1.196 & 1.345 \\
\hline T11 & 1.434 & 1.407 & 1.397 & 1.366 & 1.340 & 1.321 & 1.295 & 1.261 & 1.243 & 1.219 & 1.191 & 1.316 \\
\hline T12 & 1.171 & 1.147 & 1.132 & 1.104 & 1.085 & 1.066 & 1.038 & 1.011 & 0.987 & 0.964 & 0.944 & 1.059 \\
\hline T13 & 1.156 & 1.135 & 1.120 & 1.098 & 1.055 & 1.043 & 1.018 & 0.988 & 0.963 & 0.941 & 0.926 & 1.040 \\
\hline Mean & 1.286 & 1.255 & 1.238 & 1.208 & 1.178 & 1.162 & 1.137 & 1.109 & 1.082 & 1.055 & 1.030 & 1.158 \\
\hline S.Em \pm & 0.023 & 0.006 & 0.002 & 0.001 & 0.001 & 0.001 & 0.001 & 0.001 & 0.001 & 0.001 & 0.001 & \\
\hline CD (0.05P) & 0.066 & 0.016 & 0.005 & 0.002 & 0.002 & 0.002 & 0.002 & 0.002 & 0.002 & 0.002 & 0.002 \\
\hline CV $(\%)$ & 3.058 & 0.772 & 0.229 & 0.091 & 0.104 & 0.077 & 0.070 & 0.080 & 0.080 & 0.082 & 0.084 \\
\hline Conf
\end{tabular}

T1 - Control, T2 - ZnO NPs @ 500 mg, T3 - ZnO NPs @ 750 mg, T4 - ZnO B @ 750 mg, T5 - ZnO B @ 1000 mg, T6 - Ag NPs @ 250 mg, T7 - Ag NPs@ 500 mg, T8 - Ag B @ 500 mg, T9 - Ag B @ 750 mg, T10 - SiO2 NPs@ 250 mg, T11 - SiO2 NPs @ 500 mg, T12 - SiO2 B @ 500 mg, T13 - SiO2 B @ $750 \mathrm{mg}$

Reduction in glucose utilization occurs in the deteriorating seeds which are reflected through lower dehydrogenase activity. From this point of view, NPs treated seeds were found to have higher TDH activity and thus might have protected seeds in improving their vigour and viability. The increased availability of micronutrients at nanoscale with increased chemical reactivity resulted in the increase in synthesis and activity of the enzymes coupled with repair of damaged vital cell organelles (Burgass and Powell, 1984) ${ }^{[3]}$ and reduced counteraction of lipid peroxidation and minimization of free radical reactions (Khanahmadi et al., 2010) ${ }^{[7]}$. These beneficial effects of nano size powders were also observed by Zheng et al. (2005) ${ }^{[25]}$ in aged seeds of spinach on improving the seed vigour. Thus, the present investigation leads to the assumption that the deterioration in the enzymatic system of seed with ageing might have been protected by the NPs by quenching free radicles (Sridhar, 2012) ${ }^{[19]}$.

\section{Conclusion}

The results of our study clearly demonstrate that seed treatment with nanoparticles is a promising technology which improves the seeds quality and storability in pigeonpea. The nanoparticles are capable of entering into seeds utilizing the cracks and crevices available on the seed coat during the imbibition. This improves the enzymatic activity and free radical scavenging system by quenching the free radicals thereby lowering the oxidative damages, eventually promoting viability and vigour of seeds. Among the treatment combination, SiO2 NPs @ $250 \mathrm{mg}$ was found to be superior in maintaining or enhancing the storability of pigeonpea seeds.

\section{References}

1. Abdul-Baki AA, Anderson JD. Vigour determination of soybean seeds by multiple criteria. Crop Sci 1973;13:630-633.

2. Basu RN. An appraisal of research on wet and dry physiological seed treatments and their applicability with special reference to tropical and sub-tropical countries. Seed Sci. \& Technol 1994;22:107-126.
3. Burgass RW, Powell AA. Evidence for repair processes in the invigoration of seeds by hydration. Ann. Bot 1984;53:753-757.

4. Chinnamuthu CR, Boopathi P. Nanotechnology and agroecosystem. Madras Agric. J 2009;96(1-6):17-31.

5. Delouche JC, Baskin CC. Accelerated ageing technique for predicting the relative storability of seed lots. Seed Sci. \& Technol 1973;1:427-452.

6. ISTA. International Rules for Seed Testing. Seed Sci. \& Technol 2013;27:25-30.

7. Khanahmadi $\mathrm{M}$, Hezazadeh $\mathrm{S}$, Taran $\mathrm{M}$. In vitro antimicrobial and antioxidant properties of Smyrnium cordifolium Boiss. (Umbelliferae) extract. Asian J Plant Sci 2010;9:99-103.

8. Khot LR, Sankaran S, Maja JM, Ehsani R, Schuster EW. Applications of nanomaterials in agricultural production and crop protection: A review. Crop Prot 2012;35:64-70.

9. Kittock DL, Law AG. Relationship of seedling vigour, respiration and tetrazolium chloride reduction by germination of wheat seeds. Agron. J 1968;60:286-288.

10. Korishettar P, Vasudevan SN, Shakuntala NM, Doddagoudar SR, Hiregoudar S, Kisan B. Influence of seed polymer coating with $\mathrm{Zn}$ and Fe nanoparticles on storage potential of pigeonpea seeds under ambient conditions. J Appl. Natural Sci 2017;9(1):186-191.

11. Krishna Shyla K, Natarajan N. Customizing Zinc oxide, Silver and Titanium dioxide nanoparticles for enhancing groundnut seed quality. Indian $\mathbf{J}$ Sci. Technol 2014;7(9):1380-1385.

12. Pandey AG, Sharda S, Sanjay P, Raghvendra SY. Application of $\mathrm{ZnO}$ nanoparticles in influencing the growth rate of Cicer arietinum. J Expt. Nano. sci 2010;5(6):488-497.

13. Panse VG, Sukhatme PV. Statistical Methods for Agricultural Workers. ICAR Publication, New Delhi 1985, P359.

14. Sahebi M, Hanafi MM, Siti NAA, Rafii MY, Azizi P, Tengoua FF, Mayz A, Azw AJN, Shabanimofrad M. Importance of silicon and mechanisms of biosilica formation in plants. Biomed. Res. Int 2015;15:1-16. 
15. Sedghi M, Hadi M, Toluie SG. Effect of nano zinc oxide on the germination of soybean seeds under drought stress. Ann. West Uni. Timis. Oara. Ser. Biol 2013;2:73-78.

16. Senthilkumar S. Customizing nanoparticles for the maintanence of seed vigour and viability in Blackgram (Vigna mungo) cv. VBN 4., M.Sc. (Agri.) Thesis submitted to Tamil Nadu Agricultural University, Coimbatore (India) 2011.

17. Sharma P, Deepesh B, Zaidi MGH, Pardha SP, Khanna PK, Sandeep A. Silver nanoparticle-mediated enhancement in growth and antioxidant status of Brassica juncea. Appl. Biochem. Biotechnol 2012;167:2225-2233.

18. Shi Y, Zhang Y, Yao H, Wu J, Sun H, Gong H. Silicon improves seed germination and alleviates oxidative stress of bud seedlings in tomato under water deficit stress. Plant Physiol. Biochem 2014;78:27-36.

19. Sridhar C. Effect of nanoparticles for the maintenance of tomato seed vigour and viability. M.Sc. (Agri.) Thesis, TNAU, Coimbatore (India) 2012.

20. Surabhi VK, Rame Gowda, Nethra N. Standardization of Seed Treatment Protocol with Nanoparticles for Enhancing Seed Quality in Pigeonpea. Mysore J Agric. Sci 2018;52(3):588-596.

21. Suriyaprabha R, Karunakaran G, Yuvakkumar R, Rajendran V, Kannan N. Silica nanoparticles for increased silica availability in maize (Zea mays L.) seeds under hydroponic conditions. Curr. Nanosci 2012;8:902908.

22. Tahir M, Rahmatullah A, Aziz T, Ashraf M. Wheat genotypes differed significantly in their response to silicon nutrition under salinity stress. J Plant Nutr 2010;33:1658-1671.

23. Tejaswini KS, Kurnalliker VK, Shakuntala NM, Macha SI, Khan H, Hiregoudar S. Studies on efficacy of nanoparticles in improving seed physiological parameters in groundnut (Arachis hypogaea L.). Int. J Chem 2019;7(5):1786- 1791 .

24. Tiwari AK, Shivhare AK. Annual Progress Report. Govt. of India, Ministry of Agri. \& Farmers Welfare, Directorate of Pulses Development, Bhopal, Madhya Pradesh 2018, P1-190.

25. Zheng L, Hong F, Lu S, Liu C. Effect of nano-TiO2 on strength of naturally aged seeds and growth of spinach. Biol. Trace Elem. Res 2005;106:279-297. 